(1)

CrossMark

\title{
Pathogenesis of COVID-19 from a cell biology perspective
}

\author{
Robert J. Mason \\ Affiliation: National Jewish Health, Dept of Medicine, Denver, CO, USA.
}

Correspondence: Robert J. Mason, National Jewish Health, Dept of Medicine, 1400 Jackson Street, Denver, CO 80206, USA. E-mail: masonbanjhealth.org

@ERSpublications

COVID-19 can be understood by the region of the lung that is infected. Mild disease will be confined to the conducting airways and severe disease will involve the gas exchange portion of the lung. https:// bit.ly/2vGndRQ

Cite this article as: Mason RJ. Pathogenesis of COVID-19 from a cell biology perspective. Eur Respir J 2020; 55: 2000607 [https://doi.org/10.1183/13993003.00607-2020].

\section{Introduction}

Coronavirus disease 2019 (COVID-19) is a major health concern and can be devastating, especially for the elderly. COVID-19 is the disease caused by the SARS-CoV-2 virus. Although much is known about the mortality of the clinical disease, much less is known about its pathobiology. Although details of the cellular responses to this virus are not known, a probable course of events can be postulated based on past studies with SARS-CoV. A cellular biology perspective is useful for framing research questions and explaining the clinical course by focusing on the areas of the respiratory tract that are involved. Based on the cells that are likely infected, COVID-19 can be divided into three phases that correspond to different clinical stages of the disease [1].

\section{Stage 1: Asymptomatic state (initial 1-2 days of infection)}

The inhaled virus SARS-CoV-2 likely binds to epithelial cells in the nasal cavity and starts replicating. ACE2 is the main receptor for both SARS-CoV2 and SARS-CoV [2, 3]. In vitro data with SARS-CoV indicate that the ciliated cells are primary cells infected in the conducting airways [4]. However, this concept might need some revision, since single-cell RNA indicates low level of ACE2 expression in conducting airway cells and no obvious cell type preference [5]. There is local propagation of the virus but a limited innate immune response. At this stage the virus can be detected by nasal swabs. Although the viral burden may be low, these individuals are infectious. The RT-PCR value for the viral RNA might be useful to predict the viral load and the subsequent infectivity and clinical course. Perhaps super spreaders could be detected by these studies. For the RT-PCR cycle number to be useful, the sample collection procedure would have to be standardised. Nasal swabs might be more sensitive than throat swabs.

\section{Stage 2: Upper airway and conducting airway response (next few days)}

The virus propagates and migrates down the respiratory tract along the conducting airways, and a more robust innate immune response is triggered. Nasal swabs or sputum should yield the virus (SARS-CoV-2) as well as early markers of the innate immune response. At this time, the disease COVID-19 is clinically manifest. The level of CXCL10 (or some other innate response cytokine) may be predictive of the subsequent clinical course [6]. Viral infected epithelial cells are a major source of beta and lambda interferons [7]. CXCL10 is an interferon responsive gene that has an excellent signal to noise ratio in the 
alveolar type II cell response to both SARS-CoV and influenza $[8,9]$. CXCL10 has also been reported to be useful as disease marker in SARS $[6,10]$. Determining the host innate immune response might improve predictions on the subsequent course of the disease and need for more aggressive monitoring.

For about $80 \%$ of the infected patients, the disease will be mild and mostly restricted to the upper and conducting airways [1]. These individuals may be monitored at home with conservative symptomatic therapy.

\section{Stage 3: Hypoxia, ground glass infiltrates, and progression to ARDS}

Unfortunately, about $20 \%$ of the infected patients will progress to stage 3 disease and will develop pulmonary infiltrates and some of these will develop very severe disease. Initial estimates of the fatality rate are around $2 \%$, but this varies markedly with age [1]. The fatality and morbidity rates may be revised once the prevalence of mild and asymptomatic cases is better defined. The virus now reaches the gas exchange units of the lung and infects alveolar type II cells. Both SARS-CoV and influenza preferentially infect type II cells compared to type I cells $[11,12]$. The infected alveolar units tend to be peripheral and subpleural $[13,14]$. SARS-CoV propagates within type II cells, large number of viral particles are released, and the cells undergo apoptosis and die (figure 1) [8]. The end result is likely a self-replicating pulmonary toxin as the released viral particles infect type II cells in adjacent units. I suspect areas of the lung will likely lose most of their type II cells, and secondary pathway for epithelial regeneration will be triggered. Normally, type II cells are the precursor cells for type I cells. This postulated sequence of events has been shown in the murine model of influenza pneumonia $[15,16]$. The pathological result of SARS and COVID-19 is diffuse alveolar damage with fibrin rich hyaline membranes and a few multinucleated giant cells [17, 18]. The aberrant wound healing may lead to more severe scarring and fibrosis than other forms of ARDS. Recovery will require a vigorous innate and acquired immune response and epithelial regeneration. From my perspective, similar to influenza, administrating epithelial growth factors such as KGF might be detrimental and might increase the viral load by producing more ACE2 expressing cells [19]. Elderly individuals are particularly at risk because of their diminished immune response and reduced ability to repair the damaged epithelium. The elderly also have reduced mucociliary clearance, and this may allow the virus to spread to the gas exchange units of the lung more readily [20].

There are significant knowledge gaps in the pathogenesis of COVID-19 that will be filled in over the next few months. I based my comments on the assumption that viral entry by SARS-CoV-2 will be the same as SARS-CoV. We do not know if there are alternate receptors for viral entry. CD209L is an alternative receptor for SARS-CoV [21]. We await detailed studies on infection and the innate immune response of differentiated primary human lung cells. The apical cilia on airway cells and microvilli on type II cells may be important for facilitating viral entry.

In conclusion, COVID-19 confined to the conducting airways should be mild and treated symptomatically at home. However, COVID-19 that has progressed to the gas exchange units of the lung must be
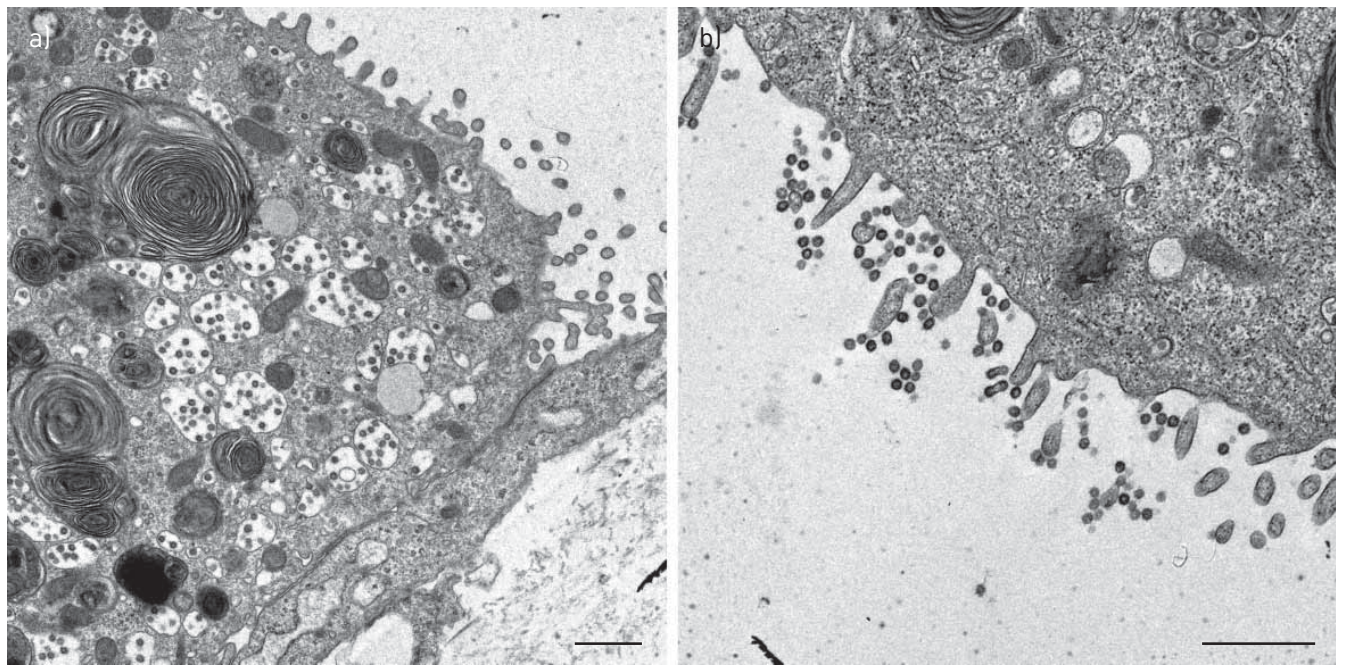

FIGURE 1 Human alveolar type II cells infected with SARS-CoV. Human type II cells were isolated, cultured in vitro, and then infected with SARS-CoV. Viral particles are seen in double membrane vesicles in the type II cells (a) and along the apical microvilli (b). Reproduced with permission from the American Thoracic Society [8]. 
monitored carefully and supported to the best of our ability, as we await the development and testing of specific antiviral drugs.

Conflict of interest: R.J. Mason has nothing to disclose.

\section{References}

1 Wu Z, McGoogan JM. Characteristics of and important lessons from the coronavirus disease 2019 (COVID-19) outbreak in China: summary of a report of 72314 cases from the Chinese Center for Disease Control and Prevention. JAMA 2020; in press.

2 Wan Y, Shang J, Graham R, et al. Receptor recognition by novel coronavirus from Wuhan: An analysis based on decade-long structural studies of SARS. J Virol 2020; 94: e00127-20.

3 Hoffmann M, Kleine-Weber H, Schroeder S, et al. SARS-CoV-2 cell entry depends on ACE2 and TMPRSS2 and is blocked by a clinically proven protease inhibitor. Cell 2020; in press.

4 Sims AC, Baric RS, Yount B, et al. Severe acute respiratory syndrome coronavirus infection of human ciliated airway epithelia: role of ciliated cells in viral spread in the conducting airways of the lungs. $J$ Virol 2005; 79: 15511-15524.

5 Reyfman PA, Walter JM, Joshi N, et al. Single-cell transcriptomic analysis of human lung provides insights into the pathobiology of pulmonary fibrosis. Am J Respir Crit Care Med 2019; 199: 1517-1536.

6 Tang NL, Chan PK, Wong CK, et al. Early enhanced expression of interferon-inducible protein-10 (CXCL-10) and other chemokines predicts adverse outcome in severe acute respiratory syndrome. Clin Chem 2005; 51: $2333-2340$.

7 Hancock AS, Stairiker CJ, Boesteanu AC, et al. Transcriptome analysis of infected and bystander type 2 alveolar epithelial cells during influenza A virus infection reveals in vivo Wnt pathway downregulation. J Virol 2018; 92: e01325-18.

8 Qian Z, Travanty EA, Oko L, et al. Innate immune response of human alveolar type II cells infected with severe acute respiratory syndrome-coronavirus. Am J Respir Cell Mol Biol 2013; 48: 742-748.

9 Wang J, Nikrad MP, Phang T, et al. Innate immune response to influenza A virus in differentiated human alveolar type II cells. Am J Respir Cell Mol Biol 2011; 45: 582-591.

10 Rockx B, Baas T, Zornetzer GA, et al. Early upregulation of acute respiratory distress syndrome-associated cytokines promotes lethal disease in an aged-mouse model of severe acute respiratory syndrome coronavirus infection. J Virol 2009; 83: 7062-7074.

11 Mossel EC, Wang J, Jeffers S, et al. SARS-CoV replicates in primary human alveolar type II cell cultures but not in type I-like cells. Virology 2008; 372: 127-135.

12 Weinheimer VK, Becher A, Tonnies M, et al. Influenza A viruses target type II pneumocytes in the human lung. J Infect Dis 2012; 206: 1685-1694.

$13 \mathrm{Wu}$, Wu X, Zeng W, et al. Chest CT findings in patients with corona virus disease 2019 and its relationship with clinical features. Invest Radiol 2020; in press.

14 Zhang S, Li H, Huang S, et al. High-resolution CT features of 17 cases of corona virus disease 2019 in Sichuan province, China. Eur Respir J 2020; in press.

15 Kumar PA, Hu Y, Yamamoto Y, et al. Distal airway stem cells yield alveoli in vitro and during lung regeneration following H1N1 influenza infection. Cell 2011; 147: 525-538.

16 Yee M, Domm W, Gelein R, et al. Alternative progenitor lineages regenerate the adult lung depleted of alveolar epithelial type 2 cells. Am J Respir Cell Mol Biol 2017; 56: 453-464.

$17 \mathrm{Gu}$ J, Korteweg C. Pathology and pathogenesis of severe acute respiratory syndrome. Am J Pathol 2007; 170: $1136-1147$.

18 Xu Z, Shi L, Wang Y, et al. Pathological findings of COVID-19 associated with acute respiratory distress syndrome. Lancet Respir Med 2020; 8: 420-422.

19 Nikolaidis NM, Noel JG, Pitstick LB, et al. Mitogenic stimulation accelerates influenza-induced mortality by increasing susceptibility of alveolar type II cells to infection. Proc Natl Acad Sci USA 2017; 114: E6613-E6622.

20 Ho JC, Chan KN, Hu WH, et al. The effect of aging on nasal mucociliary clearance, beat frequency, and ultrastructure of respiratory cilia. Am J Respir Crit Care Med 2001; 163: 983-988.

21 Jeffers SA, Tusell SM, Gillim-Ross L, et al. CD209L (L-SIGN) is a receptor for severe acute respiratory syndrome coronavirus. Proc Natl Acad Sci USA 2004; 101: 15748-15753. 